\title{
CAMBIOS Y PERMANENCIAS DE LA tecnología constructiva DE LA GASA MAYA EN MÉRIDA, YUCATÁN
}

Changes and continuities of mayan house's constructive technology at Merida, Yucatan

\begin{tabular}{|c|c|}
\hline \multicolumn{2}{|c|}{ Manuel Arturo Román-Kalisch*, Aniela Piñón-Jiménez ** } \\
\hline $\begin{array}{r}\text { Fecha de recibido: } \\
4 \text { agosto } 2018 \\
\text { Fecha de aceptado: } \\
12 \text { noviembre } 2018\end{array}$ & $\begin{array}{l}\text { *Universidad Autónoma de Yucatán, México } \\
\text { maromankog@gmail.com } \\
\text { ** Universidad Cuauhtémoc, Campus Querétaro, México } \\
\text { arq.anielapj@gmail.com }\end{array}$ \\
\hline
\end{tabular}

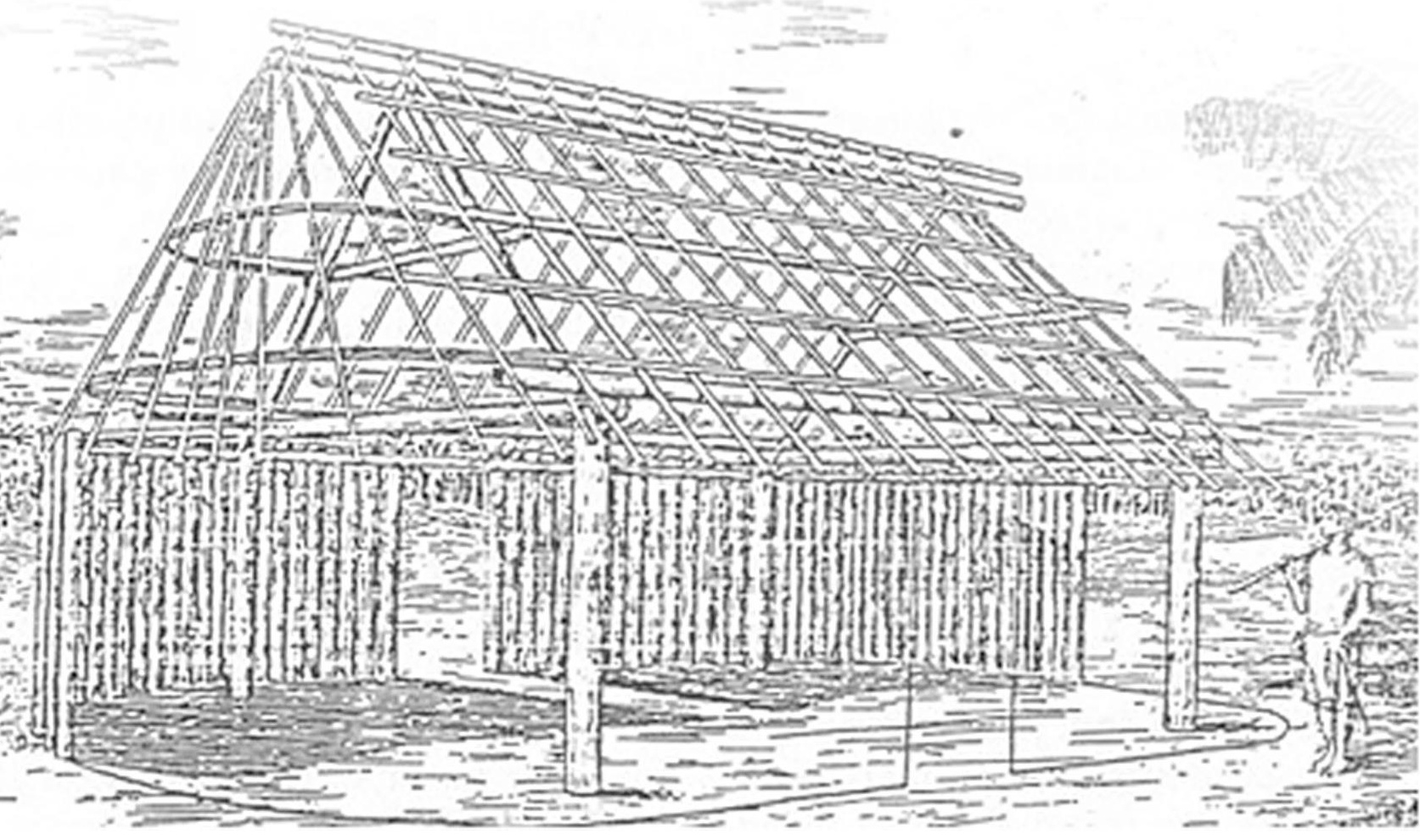


RESUMEN. Una de las características que identifica a la casa maya es la tecnología constructiva ancestral que ha sobrevivido hasta nuestros días. Esta tecnología tradicional contiene componentes importantes, como la utilización de materiales del medio ambiente natural y el conocimiento constructivo heredado. Sin embargo, desde el último tercio del siglo xx hasta el día de hoy, la vivienda maya y su tecnología constructiva se han visto afectados por diversos factores que han ocasionado cambios en su estado de conservación y en los componentes constructivos. El objetivo de este artículo es plantear los cambios y permanencias tecnológicos de las casas mayas de las comisarías en el municipio de Mérida, propiciados por factores sociales, ambientales, económicos y políticos que han dado como resultado una tipología constructiva que la caracteriza en la actualidad. Se efectuó un estudio técnico-constructivo para caracterizar a las viviendas mayas supervivientes de la zona de estudio, así mismo, se efectuaron entrevistas con sus habitantes para obtener datos sobre la antigüedad de la vivienda, constructores y transmisión del conocimiento constructivo. Se observó que las viviendas de las comisarías de la zona norte presentaron más cambios tecnológicos que las del sur y una constante fue el fuerte deterioro de las viviendas por la falta de mantenimiento y abandono.

Palabras clave: arquitectura vernácula, cambio cultural, políticas públicas, tecnología constructiva.

\begin{abstract}
A feature that identifies the Mayan House is the ancestral constructive technology that has survived to the present day. This traditional technology contains important components, such as the use of materials of the natural environment and inherited constructive knowledge. Since the last third of the 2oth century until today, however, the Mayan housing and its constructive technology have been affected by various factors that have resulted in changes in their state of conservation and building components. The objective of this work is establish changes and continuities technological in Mayan houses of commissaryships in the municipality of Mérida, brought about by social, environmental, economic, and political factors that have resulted in a typology constructive that characterizes it today. A technical-constructive study was conducted to characterize Mayan housing survivors of the study area. Likewise, interviews were conducted with its inhabitants to obtain data on the age of the housing, builders and the constructive knowledge transfer. It was observed that dwellings of the commissaryships of the North had more technological changes than those in the South and a constant was the strong deterioration of dwellings by the lack of maintenance and neglect.
\end{abstract}

Key words: constructive technology, cultural change, public policies, vernacular architecture.

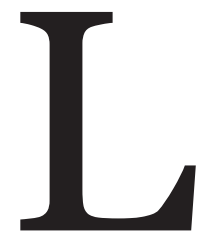

a casa maya está inmersa en una problemática social con muchas aristas que han ido deteriorando su imagen cultural y tecnológica, particularmente, en lo que respecta a la sustitución de algunos materiales de construcción tradicionales de la vivienda maya por materiales industrializados y que tiene que ver con la economía familiar, un nuevo modo de pensar de sus habitantes y la seguridad física. El solar maya es considerado como una unidad autosuficiente por las actividades realizadas en él, como las domésticas, agrícolas y de habitación. La casa maya había sido construida ancestralmente con los materiales que ofrecía el medio ambiente natural, como piedra, madera y palmas de huano; materiales que conformaron sus particularidades física, tecnológica y cultural. Sin embargo, desde hace algunas décadas, se ha presentado un proceso de sustitución de estos materiales por otros de carácter industrial que no ofrecen, en primera instancia, las mismas condiciones de confort ambiental al interior de la vivienda y demeritan sustancialmente su espacialidad y volumetría debido a sus características físicas y particulares de producción. El empleo de estos materiales se debe en parte, a la disponibilidad en el mercado de la construcción, costos accesibles y durabilidad, y, a la donación por medio de las acciones de apoyo social de organismos públicos y privados, y por otra, a la escasez y encarecimiento de las especies que proveen las hojas para cubrir las techumbres.

El abandono y el cambio de uso están asociados a la falta de seguridad ocasionada por los fenómenos hidrometeorológicos que pueden llegar afectar de manera importante a la estructura de la vivienda, a la dotación de viviendas construidas con materiales industrializados a través de las acciones de apoyo social de organismos públicos, así como a una creciente tendencia de los pobladores mayas a 


\section{La CASA MAYA} se encuentra inmersa en una PROBLEMÁTICA SOCIAL

con muchas aristas que han ido deteriorando SU IMAGEN CULTURAL Y TECNOLÓGICA.

incorporarse a la globalidad cultural, en detrimento de sus costumbres ancestrales.

La casa maya tradicional fue una respuesta a las condicionantes del medio físico y también representó la expresión de los objetivos, aspiraciones, medios y defensas de la sociedad rural. Sin embargo, "la modernización e industrialización del país trajo como consecuencia un flujo migratorio del campo a las ciudades, la producción de la arquitectura vernácula se vio condicionada y determinada por una compleja red de interacciones entre la cultura urbana y la rural, dicho intercambio cultural no fue equilibrado, la mayoría de las veces sucedió una relación asimétrica a favor de la cultura urbana" (López Morales, 1993: 12-13). De esta manera, la vivienda vernácula maya y sus elementos culturales propios, como la tradición constructiva, los sistemas y materiales tradicionales de construcción se vieron inmersos en un proceso de cambio cultural y tecnológico. Barrera (1981: 294) explica que "la casa rural y la comunidad son capaces de responder, tanto a las condiciones climáticas y ecológicas como a distintos factores propios y externos, relacionados con el medio económico, social y cultural, y ambos actores pueden cambiar gradualmente en el mismo lugar y con el paso del tiempo, así como en diferentes lugares de manera sincrónica”.

Los factores económicos pueden determinar y condicionar el cambio tecnológico de la casa maya. "Cuando los grupos rurales tienen una economía de autoconsumo, las casas tradicionales o vernáculas siguen siendo construidas con los materiales regionales y las técnicas ancestrales, la incorporación de materiales industrializados dependerá de la capacidad de acumular excedentes destinados para su adquisición" (Manual para la construcción de la vivienda rural, 1981: 14). Pérez Medina (1993: 44) explica que las tendencias a la modificación de las casas mayas hacia otros modelos arquitectónicos y tecnológicos, no sólo dependen de las condiciones económicas de las comunidades, sino que están relacionados con factores ideológicos, traducidos en nuevos intereses y valores que determinan el carácter de las viviendas, principalmente hacia los modelos de casas construidas con materiales industrializados, y con factores políticos en la construcción de este tipo de viviendas con ayudas gubernamentales.

Bolio (2016: 69-70) denuncia el alarmante ritmo de crecimiento de la superficie urbana en la Zona Metropolitana de Mérida (zMM), en donde se ubican las comisarías estudiadas (mapa 1), que provoca la devastación de la selva baja y el abandono de zonas agrícolas, y las presiones que ejerce el mercado inmobiliario para ocupar áreas agrícolas o forestales, trayendo como consecuencia el aumento de las temperaturas promedio, la falta de lluvias y la escases de la vegetación. Afectando a las especies vegetales y madereras con las que se construyen y mantienen las casas mayas en la zona de estudio.

Q Ubicación de Comisaría sin presencia actual de casa maya

Ubicación de Comisaría con presencia actual de casa maya

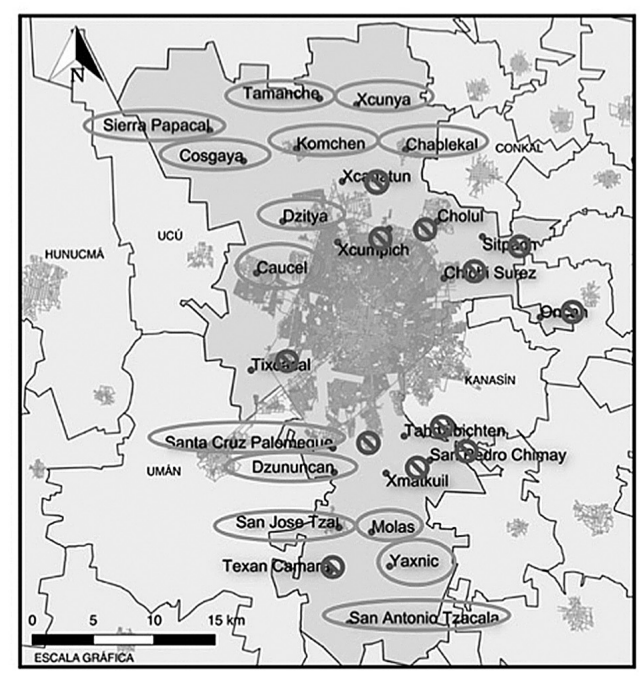

MAPA 1. UBICACIÓN DE COMISARÍAS QUE ALBERGAN ACTUALMENTE LAS CASAS MAYAS, FUENTE: (PIÑÓN JIMÉNEZ, A., 2017: 79) 
Otros aspectos de esta problemática son, por una parte, la falta de una normatividad oficial en la que se establezcan reglamentos que propicien la construcción y reparación de las techumbres de las casas mayas con materiales perecederos tradicionales y desalienten el uso de los materiales industrializados. Y por otra, el desinterés de las instituciones públicas y privadas por establecer campañas de concientización a los propietarios de las viviendas mayas para preservarlas con materiales regionales tradicionales.

En estudios recientes sobre esta problemática de cambio y sustitución tecnológica, y relacionada con la pérdida de los saberes constructivos, se tiene que "la mayoría de los habitantes de las casas mayas no conocen el procedimiento constructivo ni participaron en la construcción de su casa. Sólo algunos tenían idea de los materiales naturales que se requerían y muy pocos habían sido instruidos por sus padres o abuelos en la práctica constructiva" (Piñón, 2017: 116). Todas estas circunstancias y factores contribuyen al desarrollo de un proceso de adecuación o adaptación cultural y tecnológica de la casa maya.

\section{SISTEMA,PROCEDIMIENTOYMATERIALESDE CONSTRUCCIÓN GENÉRICOS ${ }^{5}$}

La casa maya exhibe un sistema estructural y constructivo tradicional configurado por una estructura portante, una envolvente baja horizontal de madera y, una envolvente superior inclinada vegetal. Todos los elementos de madera de la estructura portante tienen un trabajo estructural. El cuerpo bajo de la estructura portante está configurado por soportes verticales llama-

5 El sistema y procedimientos constructivos detallados de la casa maya tienen implícita una fuerte carga simbólica, ambos aspectos no forman parte de los objetivos de este trabajo, únicamente se efectúa una descripción genérica del sistema y procedimiento constructivo en este apartado. Tanto las relaciones simbólicas como el proceso constructivo detallado están muy bien expresados, entre otros autores, por Sánchez Suárez, A., García Quintanilla, A. y Eastmond Spencer, A. (2014: 57-88) y Rivas Gutiérrez, D. (2012). dos horcones. Cada horcón o noj okom reciben las cargas correspondientes al peso de la estructura y el recubrimiento de la techumbre, y se ven sometidos a esfuerzos de compresión de gran magnitud y algo de flexión. Se coloca un baloob sobre un par de horcones en el sentido transversal, cada baloob soporta las fuerzas que tienden a deformar la estructura y evita cualquier tipo de desplazamiento entre las conexiones. El taanche 'o paxná se coloca en los extremos salientes de cada baloob, en el sentido longitudinal de la estructura y se ve sometida a esfuerzos de flexión estática y a tensión. El cuerpo alto de la estructura portante está por un par de travesaños inclinados llamados tiseras y un caballete o ho'ol naj ché que presenta un alto esfuerzo en flexión estática. Cada tiseras está sujeta en su parte media por un travesaño llamado kabak que impide que aquélla se flexione en ese punto por presiones o succiones en la techumbre. Por último, la retícula inclinada que va a recibir al recubrimiento vegetal está compuesta por elementos verticales llamados wíinkil ché y por elementos horizontales o hiles, los que deben presentar mucha una gran resistencia a la flexión por la aplicación sobre ellos de presiones y succiones, producidas por los vientos que inciden directamente en la techumbre. Para conformar los ábsides de la casa, "los apoyos secundarios verticales, kulub, sostienen a dos conjuntos de maderos curvos llamado moy y kaanamoy, estos por su forma curva presentan esfuerzos altos de flexión" (Ordaz, Rodríguez y Centeno, 2014: 171-173) (figuras 1a, 1b y 1c).

\section{la CASA MAYA exhibe un sistema estructural v constructivo tradicional CONFIGURADO POR UNA ESTRUCTURA PORTANTE,}

$$
\begin{array}{r}
\text { una envolvente baja } \\
\text { horizontal de madera y, } \\
\text { UNA ENVOLVENTE SUPERIOR } \\
\text { INCLINADA VEGETAL. }
\end{array}
$$




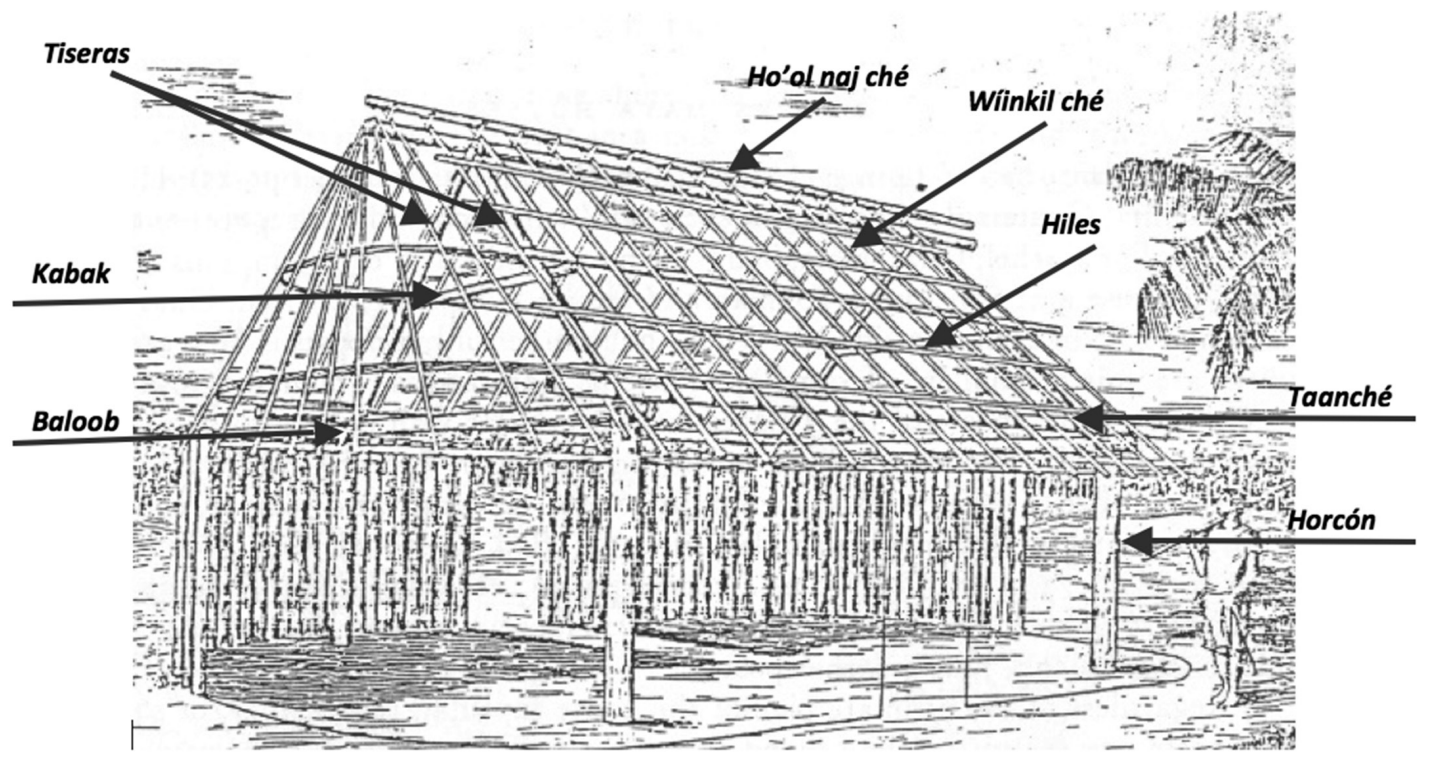

FIGURA 1A. CROQUIS PERSPECTIVO DE UNA CASA MAYA DONDE SE MUESTRAN LOS ELEMENTOS ESTRUCTURALES Y CONSTRUCTIVOS DE MADERA. FUENTE: WAUCHOPE, R. (1939).

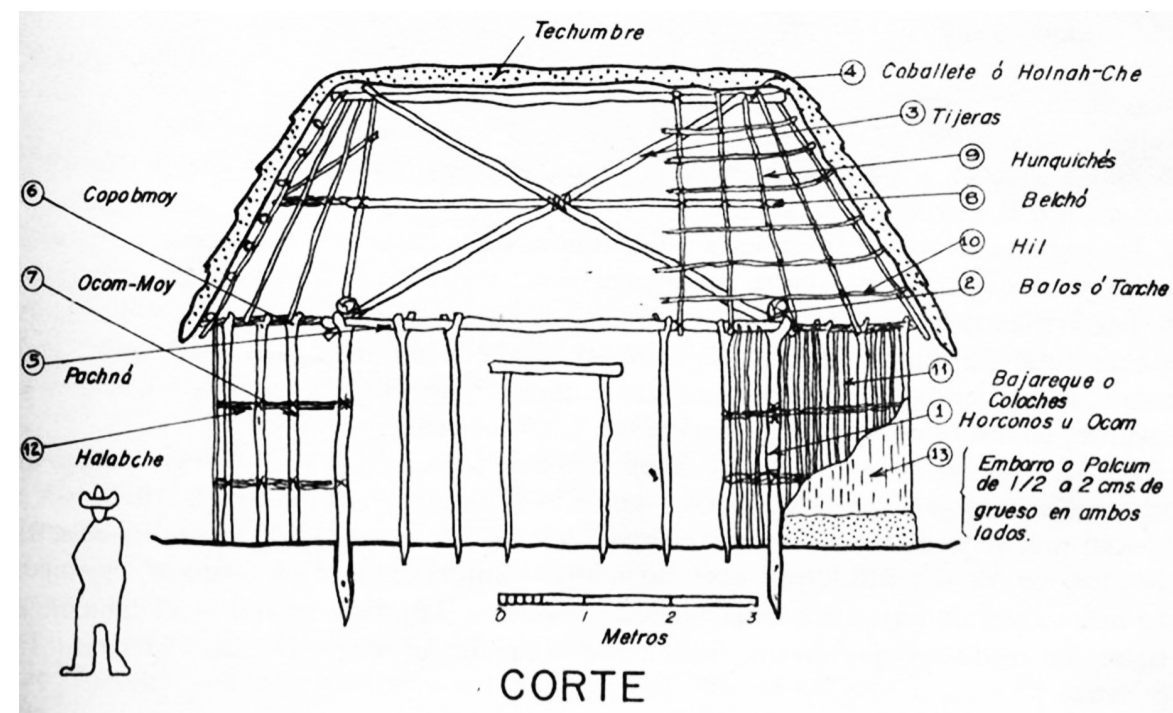

Figura 1B. CORTE dE unA CASA MAYA DONDE SE MUESTRAN LOS ElEMENTOS ESTRUCTURALES Y CONSTRUCTIVOS DE MADERA.

FUENTE: (MOYA RUBIO, V. J., 1988: 79).

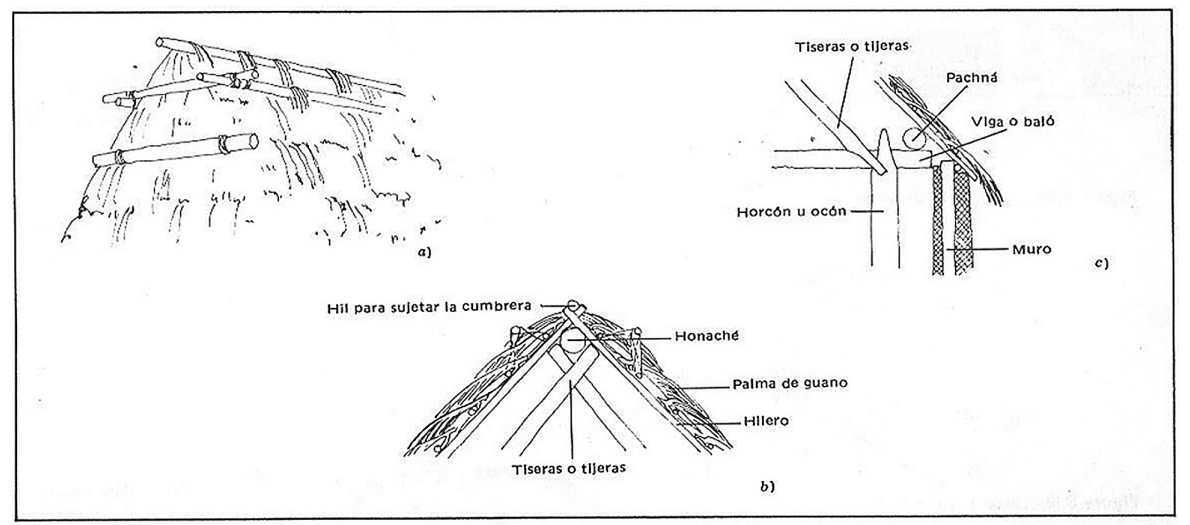

FiGURA 1C. DETALLES CONSTRUCTIVOS: AJ CUMBRERA Y REMATE, B] CORTE CUMBRERA Y REMATE, Y C] UNIONES DE ESTRUCTURA PORTANTE Y MURO. FUENTE: (LÓPEZ MORALES, F. J., 1993: 263). 
Como se puede apreciar, es un sistema constructivo que requiere de práctica para llevarse a cabo y del conocimiento de cada uno de los elementos estructurales y constructivos que intervienen en la edificación, así como de las dimensiones y tipos de madera para cada uno de estos. Los resultados preliminares de las entrevistas realizadas a los habitantes de las casas mayas en la zona de estudio, indican que la mayoría de ellos no conocen el sistema constructivo ni participaron en la obra; muy pocos tienen el conocimiento y la práctica constructiva, aprendidos de familiares y constructores independientes; sólo uno de aquellos indicó que enseñó a su hijo.

En Timul y Chablekal, Yucatán, se utilizaba la madera del árbol de chucum para horcones y balos, en lo que respecta al huano, ya no se encuentran palmas en la comunidad y tienen que obtenerlas lejos de ahí y pagarlas al dueño de los terrenos. ${ }^{6}$ Existe una gran variedad de especies maderables con las que se puede construir una casa maya, la elección del tipo de madera depende de la experiencia de los constructores y de la disponibilidad de las especies. ${ }^{7}$

Rangel (1980: 56) apunta que en los poblados donde se realizaron entrevistas a sus habitantes, "no todos poseían los conocimientos suficientes para la construcción de casas y tampoco conocían las dimensiones necesarias, por lo que las construían basándose en las dimensiones de las viviendas existentes". En Hunucmá, uno de los constructores de casas mayas y palapas, mencionó que utiliza maderas y hojas de huano de la región, así como clavos de concreto para las uniones; así mismo, comentó que ha hecho y reparado una gran cantidad de casas mayas, pero que ya tiene tiempo que no le encargan viviendas de este tipo. ${ }^{8}$ La utilización de elementos metálicos para la fijación de los elementos estructurales de madera puede ser producto de la escasez del material vegetal para hacer los amarres en las uniones de estas piezas o bien a la facilidad de la adquisición de las piezas metálicas y la rapidez para efectuar la fijación de las piezas.

Algunos autores mencionan que la extracción de las maderas para la construcción de la casa maya debe de ser durante la época seca, ya que "la madera contiene menos humedad, tendrá mayor tiempo de vida y resistirá mejor el ataque de insectos y microorganismos" (Quiroz et al., 2011: 33). Sin embargo, otros autores coinciden en que la época de corte debe ser en las épocas de lluvia; el tratamiento al que se someten las maderas principales consiste en descortezar y secar, y, únicamente, usarlas cuando estén secas (Sánchez, García y Eastmond (2014:128), Ordaz, Rodríguez y Centeno (2014:129).

\section{TIPOLOGÍA CONSTRUCTIVA}

El sistema estructural y constructivo de la casa maya tradicional presenta en la actualidad variantes constructivas en las envolventes horizontal y vertical, la primera con tres variantes: apoyos corridos de madera, piedra y materiales industrializados; y la segunda, la cubierta con recubrimientos vegetales y materiales industrializados; mientras que la estructura portante mantiene sus elementos constitutivos sin cambios fundamentales. La tipología constructiva se presenta a continuación caracterizando sus elementos constituyentes, los cuales se presentan según el procedimiento genérico de la casa maya: rodapié, estructura portante, muros y cubiertas.

\section{RODAPIÉ}

6 Comunicación oral de Nelly Canul Cauich en junio de 2010, quien habitó en su niñez una casa maya tradicional en Timul, Yucatán.

7 Para consultar con detalle las especies vegetales y maderables, así como los elementos estructurales y constructivos en que se utilizan, ver a Villers Ruíz, L., López Franco, R. M. y Barrera, A. (2014: 129-141); Ordaz Tamayo, M., Rodríguez Pérez, I. y Centeno Lara, R. (2014: 169-170); Sánchez Suárez, A., García Quintanilla, A. y Eastmond Spencer, A. (2014: 77); Eastmond, A., García Quintanilla, A. y Sánchez Suárez, A. (2014: 234-238), y Quiroz et al. (2011: 31-33).

8 Entrevista realizada al Sr. José Demetrio “Don Saboro" en septiembre de 2016.
Es un elemento corrido de mampostería que sirve para recibir al muro de bajareque y al sobresalir del nivel de terreno natural evita que la humedad suba al bajareque. El ancho del rodapié es de 30 a $40 \mathrm{~cm}$ y la altura es variable; el aparejo de la mampostería es ordinario y se colocan las piedras a hueso como el sistema de albarrada, a veces las juntas se refuerzan con mortero de cal, como se puede observar en una vivienda en Chablekal; 


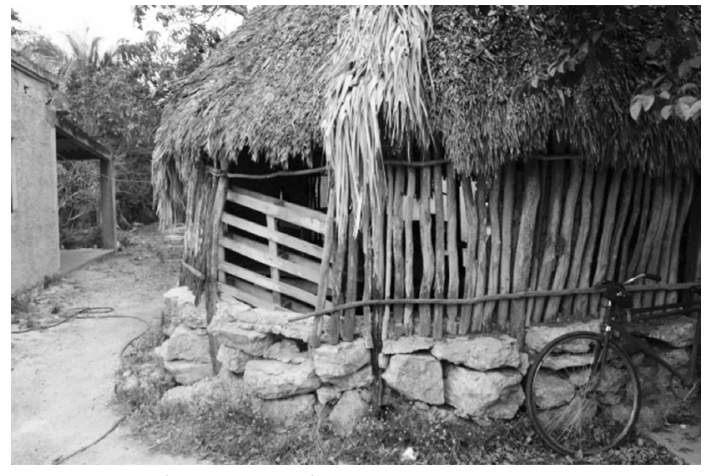

FIGURA 2. RODAPIÉ DE MAMPOSTERÍA EN UNA VIVIENDA DE CHABLEKAL.

FUENTE: (LÓPEZ MORALES, F. J., 1993: 263).

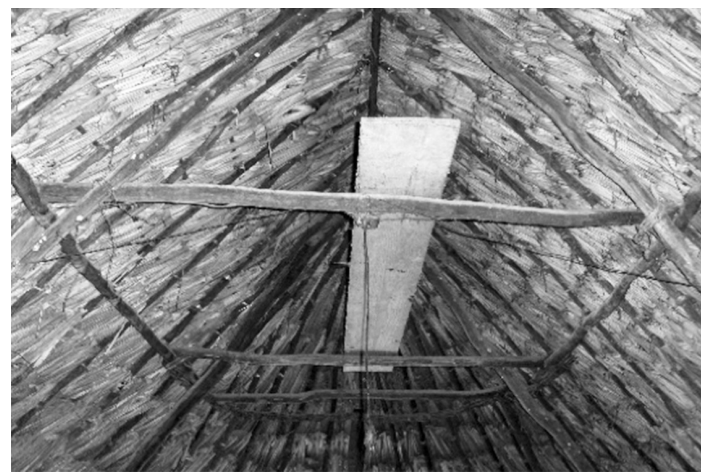

FIGURA 3. DETALLE DE TIJERAS, CUMBRERA Y ELEMENTOS RIGIDIZANTES SECUNDARIOS EN UNA VIVIENDA DE CHABLEKAL.

FUENTE: ELABORACIÓN PROPIA.

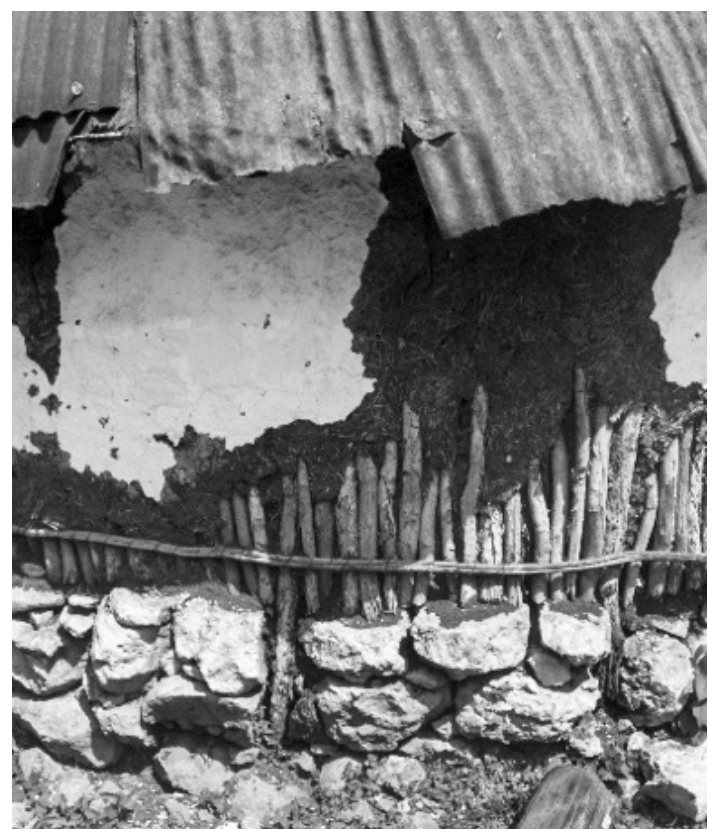

FIGURA 4. EMBARRO Y ENCALADO EN MURO DE BAJAREQUE EN UNA VIVIENDA DE SAN JOSÉ TZAL.

FUENTE: ELABORACIÓN PROPIA. se dejan espacios en el rodapié para librar a los kulub de los ábsides y a los horcones de la estructura portante (figura 2).

\section{ESTRUCTURA PORTANTE}

La parte inferior de los horcones está hincada en el terreno y, la superior, presenta una horqueta donde recibe al balo y éste a su vez recibe al taanche' o paxná, la unión de estos últimos elementos se sujetan con alambre recocido o bejuco. Los pares de tijeras se amarran a los horcones de igual manera que los anteriores elementos y éstas reciben a la cumbrera o ho'lnache'; a media altura de las tijeras se amarran los elementos longitudinales y transversales de rigidización complementaria: Kaanataanche' y kabah; sólo hubo un caso de una vivienda en Molas, con un tercer par de tijeras ubicadas al centro de la casa. En todos los casos observados, no existen los elementos de rigidización diagonal llamados alka'ch'o o belch o (figura 3).

La estructura secundaria que recibe a la cubierta vegetal o de materiales industrializados, se configura por un entramado de elementos de madera verticales y horizontales: winkiche' y hil; se ha observado que pueden ser amarrados con bejucos, sogas de henequén o con alambre recocido.

\section{MURO DE BAJAREQUE}

Este elemento sirve de envolvente horizontal corrida a la vivienda. El muro de bajareque se configura por un muro de maderas y un embarro. Cada pieza del muro de madera se llama kololché y permanece asentado en la parte inferior en el rodapié y, en la superior, está unido por su horqueta al copobmoy; este entramado de kololché se une y rigidiza por medio de los halabche, en algunos casos se componen de una o dos piezas y están amarrados con alambre recocido en lugar de bejuco. $\mathrm{Al}$ muro de madera se le aplana por fuera y por dentro 
con una mezcla de tierra vegetal roja, llamado embarro o pakluum, de grosores variables y se le aplica una pintura a la cal, a este procedimiento se le llama encalado. La falta de mantenimiento y el abandono provoca la pérdida parcial o total de los aplanados (figura 4).

\section{MURO DE MAMPOSTERÍA}

Este elemento sustituye al muro de bajareque como la envolvente horizontal de la vivienda, la provee de mayor resistencia a los vientos huracanados. El confort térmico se mantiene cuando las casas mayas tienen cubiertas de huano y muros de mampostería o de bajareque, sin embargo, “disminuye significativamente el confort térmico al interior de viviendas no vernáculas cuando las cubiertas son de láminas de cartón, aumentando la temperatura más de $5^{\circ}$ C" (Canto, Contreras y Ruíz, 2014: 119-120).

Así mismo, toma el trabajo de soporte del kulub y del copobmoy, ocasionalmente sustituye a los horcones y por lo general se encuentran exentos del eje longitudinal de estos elementos. El muro es de mampostería ordinaria con juntas rajueleadas de mortero de cal y cemento; el ancho varía de 30 a $40 \mathrm{~cm}$; en unos casos, no tiene aplanados exteriores y, en otros, está aplanado con mortero del mismo material del muro y un aplanado final liso de estuco. En algunos casos, se observa en los ábsides el desgaste de los aplanados provocado, posiblemente, por el continuo golpeo del viento (figura 5).

\section{MURO DE BLOCK}

Se observó el caso de una vivienda maya de planta absidal con muros de block de concreto vibro comprimido; dicho muro presenta las mismas funciones que el de mampostería. En uno de los ejes longitudinales, el muro se exhibe exento a los dos horcones de la estructura portante, mientras que en el otro, los horcones están embebidos en el muro y están recubiertos de concreto, como si fueran castillos armados (figura 6).

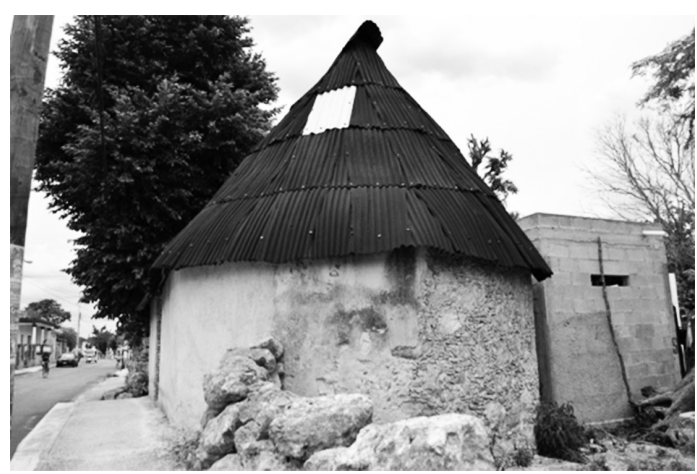

FIGURA 5. MURO DE MAMPOSTERÍA EN DONDE SE OBSERVA EL APAREJO Y EL APLANADO ENUNA VIVENDA DE MOLAS.

FUENTE: ELABORACIÓN PROPIA.

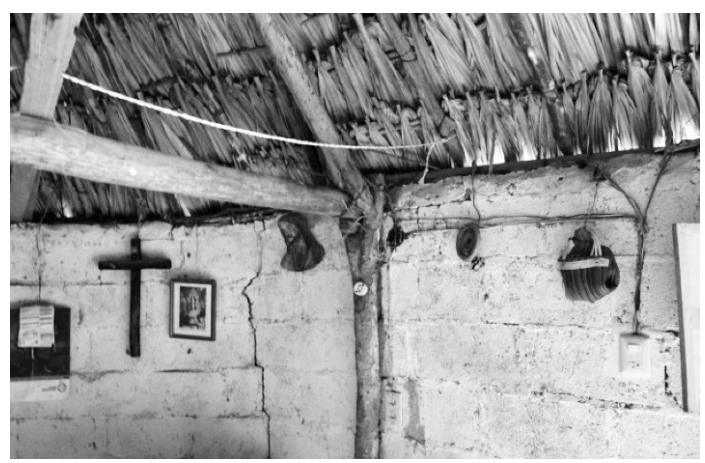

FIGURA 6. MURO DE MAMPOSTERÍA EN DONDE SE OBSERVA EL APAREJO Y EL APLANADO EN UNA VIVIENDA DE MOLAS

FUENTE: ELABORACIÓN PROPIA

\section{CUBIERTA DE HUANO}

Existen diferentes clases de palma dehuano oxa'an, que son utilizadas para cubrir las casas mayas, como Reinhardtia, Sabal mayarum, Sabal mexicana o Sabal japa. Cada palma de huano se coloca entre los hiles, de tal manera que se encajan una junta a otra en el sentido longitudinal, estando cuatrapeadas cada una de las hiladas horizontales de palmas. La cumbrera es el elemento que más daño presenta en condiciones normales de vida útil y al ser el elemento que más mantenimiento requiere, frecuentemente no se repara con las palmas de huano, sino que se sustituyen por láminas de cartón asfaltado o zinc (figura 7).

La utilización de la palma de huano permite que el aire caliente, el cual al ser más ligero, sea expulsado por los pequeños orificios que se forman entre la retícula. Por tanto, "se genera un confort térmico con temperaturas más confortables aún en los meses de mayor calor" (Canto, Contreras y Ruíz, 2014: 120). 


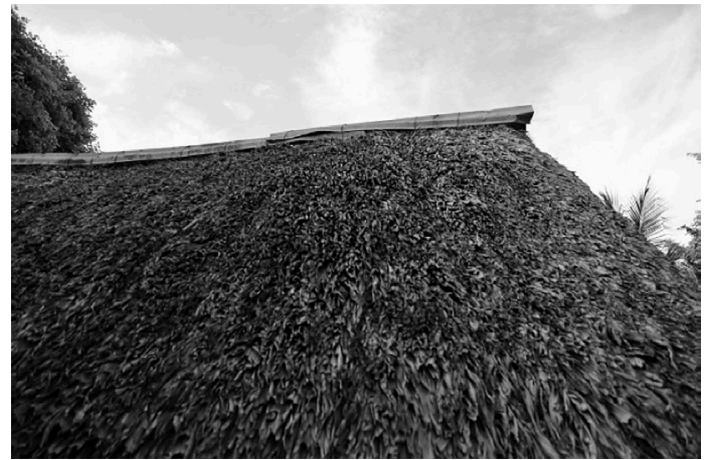

FIGURA 7. DETALLE DE CUMBRERA CON LÁMINA DE ZINC EN CUBIERTA DE HUANO EN UNA VIVIENDA DE CAUCEL.

FUENTE: ELABORACIÓN PROPIA.

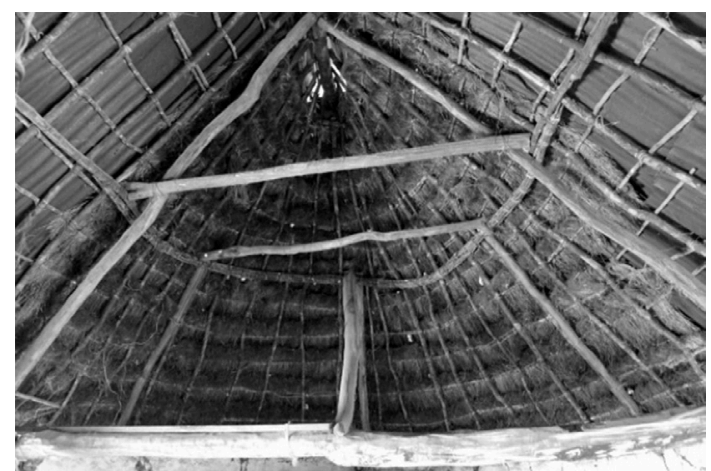

FIGURA 8. SUSTITUCIÓN PARCIAL DE LA CUBIERTA DE ZACATE CON LÁMINA DE CARTÓN ASFALTADO EN UNA VIVIENDA DE SAN JOSÉ DZAL.

FUENTE: ELABORACIÓN PROPIA.

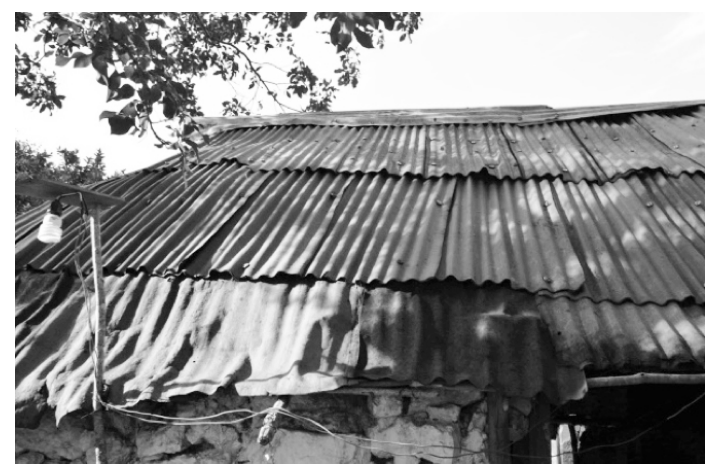

FIGURA 9. SUSTITUCIÓN TOTAL DE LÁMINA DE CARTÓN ASFALTADO EN LA CUBIERTA DE UNA VIVIENDA DE XCUNYA

FUENTE: ELABORACIÓN PROPIA.

\section{CUBIERTA DE ZACATE}

Varias casas tuvieron en un principio recubrimiento de zacate, en lengua maya, su'uk, para una denominación general, aak, para el zacate de color verde y, chak'uk, para el zacate rojo, de la sabana; al escasear el material vegetal los habitantes de las casas lo cambiaron por láminas de cartón asfaltado, como fue el caso de dos viviendas en Molas. Sin embargo, en una vivienda de San José Tzal, todavía sobrevive la cubierta de zacate a la que se ha ido cambiando por lámina de cartón asfaltado; el ábside es la parte de la cubierta que conserva todavía el recubrimiento de zacate (figura 8).

\section{CUBIERTA DE LÁMINA DE CARTÓN ASFALTADO}

Este material se ha estado utilizando en sustitución de las palmas de huano, de manera parcial, en reparaciones menores, o total, cambiando la fisonomía natural de la vivienda maya y el confort térmico del interior. Las láminas se fijan al entramado de madera a base de clavos y tapas de lata de refrescos embotellados, se van colocando de abajo hacia arriba con un cuatrapeo suficiente para evitar la entrada del agua pluvial (figura 9).

Las cubiertas con lámina de cartón asfaltado mantienen el aire caliente a diferencia del flujo de aire caliente hacia el exterior que propician las cubiertas con palma de huano o zacate. La lámina de cartón asfaltado, tanto por su composición como por su tonalidad, retiene mayor cantidad de calor y no permite que se expulse el aire caliente eficientemente, es decir, el aire caliente queda resguardado en la parte superior de la vivienda, provocando que la temperatura en el interior tenga una diferencia de más de $3^{\circ} \mathrm{C}$, respecto a las cubiertas con palma de huano (Canto, Contreras y Ruíz, 2014: 119).

\section{CUBIERTA DE LÁMINA DE ZINC}

$\mathrm{Al}$ igual que el material anterior, la lámina de zinc sustituye a la cubierta de huano de forma parcial o total e inclusive a las láminas de cartón asfaltado deterioradas, disminuyendo aún más el confort climático al interior de la vivienda. Las láminas se colocan de abajo hacia arriba y se fijan al entramado de madera con pernos metálicos (figura 10). 
En la subcomisaria de Tixcuytun se encontraron dos viviendas a las que se les sustituyó totalmente el entramado de madera y la cubierta de huano con techumbres de ferro cemento con láminas de poliestireno integradas para tratar de conservar el confort térmico al interior de las casas, sin embargo, no conservaron la altura y proporciones de la cubierta original (figura 11).

\section{CONCLUSIONES}

La escasez y progresiva desaparición de las palmas de huano y el zacate, aunado a la continua práctica de las instancias gubernamentales y privadas de dotar, a los habitantes de las poblaciones en el estado de Yucatán, de materiales industrializados (Cocom, 2016: 13), "propician el cambio y sustitución de las cubiertas con materiales vegetales, cuando lo deseable sería la promoción de la reforestación de los solares y los terrenos aledaños a las poblaciones con plantas de huano, con el fin de recuperar, para su explotación, estas especies y contribuir a reforzar la economía familiar".

A pesar de que hay una pérdida gradual de conocimiento y práctica constructiva de la casa maya, las viviendas existentes reflejan el dominio técnico y los saberes constructivos de las personas que las edificaron, y que les permiten prevalecer a pesar de la falta de algunos elementos de la parte superior de la estructura portante.

La tipología constructiva actual es el resultado del proceso de cambio y permanencia de sistemas, procedimientos y materiales utilizados en la edificación de las casas mayas. La permanencia constructiva se manifiesta en los rodapiés, muros de bahareque y de mampostería, así como la estructura portante, con ciertos faltantes y diferencias que la caracterizan.

\section{La TIPOLOGÍA CONSTRUCTIVA actual es el resultado
del proceso de cambio y
permanencia de sistemas, PROCEDIMIENTOS Y MATERIALES utilizados en la edificación DE LAS CASAS MAYAS.}

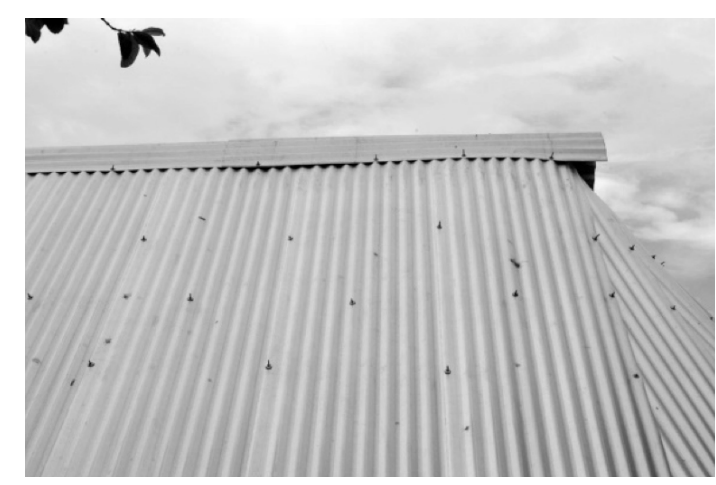

FIGURA 10. DETALLE DE CUBIERTA DE LÁMINA DE ZINC DE UNA VIVIENDA DE DZIBICHALTÚN.

FUENTE: ELABORACIÓN PROPIA

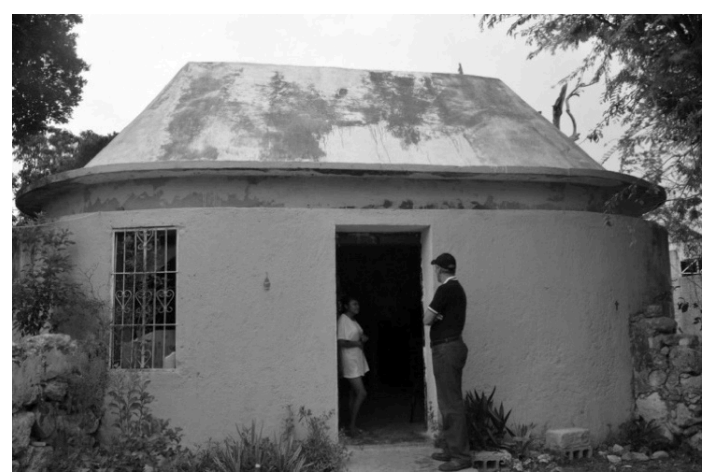

FIGURA 11. CUBiERTA DE FERRO CEMENTO EN UNA VIVIENDA DE TIXCUYTÚN. FUENTE: ELABORACIÓN PROPIA. 
La permanencia de la utilización en los muros de materiales naturales como el bahareque representa un $54 \%$ y los de mampostería representan el 38\%. Mientras que, en lo relativo al cambio tecnológico, "los materiales industrializados como el block de concreto sólo representan el 8\% del total de viviendas que hay actualmente en las comisarías de Mérida, (Piñón, 2017: 100).

El proceso de cambio y permanencia se manifiesta más en los materiales utilizados en las cubiertas de las viviendas. Existe el predominio del uso de materiales industrializados como las láminas de cartón asfáltico y la de zinc, que representa el $51 \%$ del total de las viviendas analizadas. "La permanencia del empleo de la palma de huano únicamente es del 30\%, y por último, la utilización de cubiertas combinadas con materiales vegetales y los materiales industrializados, representa el 16\%" 5 (Piñón, 2017:112).

Esta situación trae consigo afectaciones al paisaje arquitectónico y urbano de las poblaciones estudiadas, la contaminación ambiental por el fomento en el uso de los materiales industrializados, la diminución de la práctica constructiva ancestral de los saberes constructivos y la pérdida del confort climático al interior de estas viviendas. Tal problemática pone en situación de alto riesgo a la vivienda maya y a su permanencia como patrimonio vernáculo, situación que se debe de revertir con acciones de concientización y de reforzamiento social y económico tanto a nivel de pobladores como a nivel de instituciones públicas y privadas. 


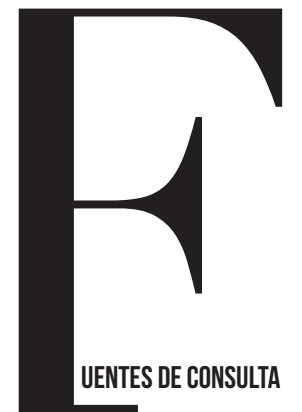

Barrera, A., López Franco, R. M. y Villers, L. (1981), "La unidad de habitación tradicional campesina y el manejo de recursos bióticos en el área maya yucatanense”, Biótica, vol. 6, núm. 3, pp. 293-323.

Bolio Osés, J. (2016), En unas cuantas manos. Urbanización neoliberal en la periferia metropolitana de Mérida, Yucatán, 2000-2014, Universidad Autónoma de Yucatán, Mérida.

Canto Cetina, R., Contreras A. y Ruiz R. (2014), "Sistemas constructivos y comportamiento térmico en el medio rural en Yucatán", Memorias xхxvII Semana Nacional de Energía Solar, Asociación Nacional de Energía Solar, Querétaro.

Cocom, L. (2016), "Apoyan a familias que carecen de vivienda digna", Por Esto, 23 de septiembre de 2016, p. 13 .

Eastmond, A., García Quintanilla A. y Sánchez Suárez A. (2014), "La casa maya y sus aportaciones hacia la sustentabilidad en Yucatán” en A. Sánchez Suárez y A. García Quintanilla (eds.), Las casa de los mayas de la península de Yucatán: historias de la maya naj, Universidad Autónoma de Yucatán, Plaza y Valdés, México.

López Morales, F. J. (1993), Arquitectura vernácula en México, $3^{\mathrm{a}}$ ed., Trillas, México.

Manual para la construcción de la vivienda rural (1981), Secretaría de Asentamientos Humanos y Obras Públicas, México.

Moya Rubio, V. J. (1988), La vivienda indígena de México y del mundo, $3^{\mathrm{a}}$ ed., Universidad Nacional Autónoma de México, México.

Ordaz Tamayo, M., Rodríguez Pérez, I. y Centeno Lara, R. (2014), "La casa maya en la zona costera del estado de Yucatán: técnica tradicional de construcción y factores de riesgo" en Sánchez Suárez. A. y A. García Quintanilla (eds.), Las casa de los mayas de la península de Yucatán: historias de la maya naj, Universidad Autónoma de Yucatán, Plaza y Valdés, México.
Pérez Medina, S. (1993), "Transformación de la vivienda rural en Yucatán: estudios de caso", Cuadernos de arquitectura de Yucatán, núm. 6, pp. 38-45.

Piñón Jiménez, A. (2017), El proceso de adecuación tecnológica de la casa maya en las comisarías del municipio de Mérida, Yucatán. Tesis de maestría. Facultad de Arquitectura de la Universidad Autónoma de Yucatán, Mérida.

Quiroz Carranza J., Cantú Gutiérrez C., Flores Quiroz F. y Herrera Vázquez M. (2011), "Vivienda tradicional maya, una arquitectura tradicional, apropiada y apropiable", Palapa, vol. VI, núm. 1(12), pp. 31-33.

Rangel, A. (1980), "El hábitat maya", Arquitectura vernácula. Cuadernos de Arquitectura y Conservación del Patrimonio Artístico, núm. 10, pp. 50-59.

Rivas Gutiérrez, D. (2012). La choza maya. Cuna y custodia de los grandes misterios y de la sabiduría de una cultura que sigue viva, Universidad Autónoma de Yucatán, México.

Sánchez Suárez, A., García Quintanilla, A. y Eastmond Spencer, A. (2014), "La construcción simbólica, formal y material de la casa maya”, en Sánchez Suárez. A. y. García Quintanilla, A. (eds.), Las casa de los mayas de la península de Yucatán: historias de la maya naj, Universidad Autónoma de Yucatán, Plaza y Valdés, México.

Villers Ruíz, L., López Franco, R. M. y Barrera, A. (2014), "La unidad de habitación tradicional campesina y el manejo de recursos bióticos en el área maya yucatanense: materiales vegetales en la habitación rural tradicional Cobá, Quintana Roo" en Sánchez Suárez. A. y García Quintanilla, A. (eds.), Las casa de los mayas de la península de Yucatán: historias de la maya naj, Universidad Autónoma de Yucatán, Plaza y Valdés, México.

Wauchope, R. (1939), Modern Maya house. A study of their archaeological significance, Carnegie Institution of Washington, Washington. 